Germany. "You will have a hard time finding a single somatic mutation that is as prevalent in this particular cancer."

Because these epigenetic changes can potentially be reversed by drugs, they are good targets for the prevention and treatment of disease. To assess the effect of epigenetic regulation on health and disease, researchers are now cataloguing epigenetic variations across the genome - or epigenome - in different tissues and at various stages of development.

\section{Chips with everything}

Cytosine is typically methylated when it is next to guanine in what is known as a $\mathrm{CpG}$ dinucleotide. Although most CpGs are methylated in mammals, some are not methylated and these are usually grouped in clusters called CpG islands. These tend to be located in the 5 regulatory regions of genes. In many cancers, these CpG islands become hypermethylated, resulting in the heritable silencing of transcription of downstream genes.

DNA methylation can be detected in several ways. One method compares what happens when genomic DNA is digested by enzymes that are either sensitive or insensitive to methylation. Another approach uses chromatin

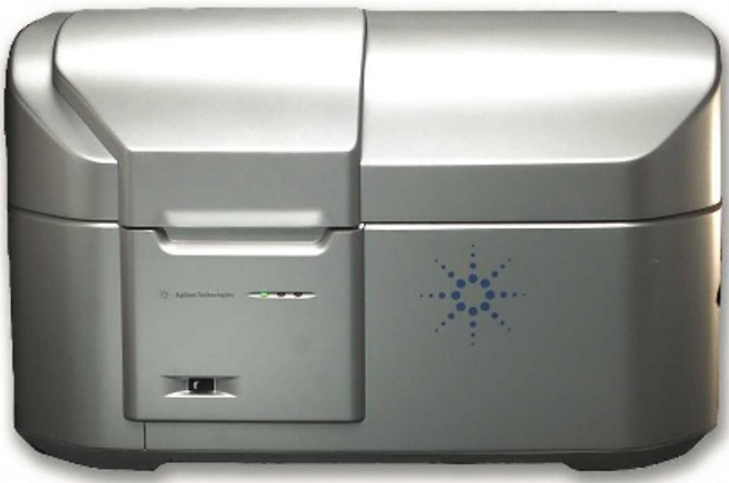

Agilent's DNA microscanner has a resolution of 2 micrometres.

What we are looking for are regions with higher densities of methylation," says Mary Harper, chief scientific officer at Genpathway in San Diego, California. "These can be regulatory regions and there are tens of thousands of these regions across the 원 genome."

ChIP-on-chip has grown in popularity with the availability of high-density arrays of contiguously tiled oligonucleotide probes. Affymetrix of Santa Clara, California, offers whole-genome tiling yeast arrays with a resolution of 5 base pairs (a total of 3.2 million probes). The company also makes human and mouse whole-genome

immunoprecipitation, or ChIP. This involves crosslinking DNA with its associated proteins and then shearing the DNA. The fragments that contain methylated cytosine are extracted by immunoprecipitation with antibodies specific for 5-methylcytosine or fragments associated with other proteins such as transcription factors or histones. The immunoprecipitated DNA is purified, amplified and labelled with a fluorescent tag. This is then applied to the surface of a DNA microarray containing a set of probes - a procedure commonly referred to as ChIP-on-chip.

"The genome is methylated at a low level everywhere, but it is more of a sprinkling. tiling arrays, each as a set of 14 arrays containing around 45 million probes at a spacing of 35 base pairs.

Along with other companies such as NimbleGen in Madison, Wisconsin, and Agilent Technologies in Santa Clara, California, Affymetrix provides several array formats for ChIP-onchip experiments. In addition to whole genome arrays, these companies sell CpG islands arrays, promoter arrays, ENCODE arrays (see 'Tackling the epigenome') or custom-made arrays. The choice of the array depends mainly on the type of experiment being done, the resolution needed and cost.

"Different arrays are not that different,"

\title{
TOOLS OF THETRADE
}

For researchers tackling the epigenome, there are two key tools: bisulphite conversion kits and ChIP-grade antibodies.

Getting complete conversion of unmethylated cytosine to uracil has been one of the main challenges for the bisulphite treatment of DNA, not least because the reaction conditions can cause the DNA to degrade. A number of companies have tackled the problem.

"There are incredibly good kits for bisulphite conversion," says Achim Plum, senior vice-president for corporate development at Epigenomics in Berlin. "If you use home brews you can make many mistakes, but with kits this is no longer an issue." QIAGEN of Venlo, the Netherlands, sells EpiTect, a kit based on Epigenomics' technology that Plum says offers nearly $100 \%$ conversion without DNA degradation. In June, QIAGEN expanded the EpiTect product line to include a standardized workflow for methylation analysis from sample collection, stabilization and purification to bisulphite conversion, and real-time endpoint

\section{PCR methylation} analysis or sequencing. Epigentek in Brooklyn, New York, also sells epigenetic products and kits for DNA methylation and histone modification. Its Methylamp DNA modification kit takes two hours to convert cytosine to uracil with more than $99 \%$ accuracy and requires only 50 picograms of starting DNA, according to Adam Li, the company's chief scientific officer. The sample can then be applied for methylation-specific PCR or a methylation array.

In June last year, Sigma-Aldrich of St Louis, Missouri, launched a number of products for epigenetic research based on Epigentek's technologies. Other providers of bisulphite conversion kits and related epigenetics products include Human Genetic Signatures in Sydney, Australia; Invitrogen of Carlsbad, California; Promega of
Epigentek's Methylamp kit takes two hours to convert cytosines to uracil.

target while it is bound to DNA, the epitope that the antibody recognizes must be available in a spatial conformation that is not obscured by the shape the protein takes while bound to DNA," explains Dana Meents, a product manager at Active Motif in Carlsbad, California. Active Motif is one of several companies now selling products that have been ChIP validated.

Abcam in Cambridge, UK, sells just over 200 ChIP-grade antibodies, many of them specific to histone post-translational modifications.

As an alternative to antibodies, the HaloCHIP system developed by Promega allows researchers to perform ChIP-on-chip experiments by cross-linking DNA to a protein of interest fused to a modified haloalkane dehalogenase tag (HaloTag). The protein-DNA complex can then be captured on a resin that recognizes the HaloTag for further microarray analysis. L.B. 